\title{
Numerical simulation of transverse jet flow field under supersonic inflow
}

\author{
LI Qian ${ }^{1, *}$, WANG Dian-kai ${ }^{1}$, YANG Yin-jun ${ }^{2}$, FANG Juan ${ }^{3}$ \\ ${ }^{1}$ State Key Laboratory of Laser Propulsion \& Application, Space Engineering University, Beijing 101416, China \\ ${ }^{2}$ Xichang Satellite Launch Center of China, Xichang Sichuan, 615606 \\ ${ }^{3}$ Jiuquan Satellite Launch Center of China, Jiuquan Gansu, 732750
}

\begin{abstract}
Transverse jet flow field under supersonic inflow is simulated numerically for studying the characteristic of fuel transverse jet and fuel mixing in scramjet combustion chamber. Comparison is performed between simulated results and the results of references and experiments. Results indicate that the CFD code in this paper is applicable for simulation of transverse jut flow field under supersonic inflow, but in order to providing more effective numerical predictive method, CFD code should be modified through increasing mesh density and adding LES module.
\end{abstract}

Key words. supersonic inflow; transverse jet; fuel mixing; numerical simulation

\section{Introduction}

With the development of aerospace technology, higher flight velocity is purchased by people, so the study of scramjet is becoming one of the key problems in the research field of hypersonic vehicle. Compared with other ramjet engines, problems exist in the mixing of air and fuel in scramjet: (1) When the inflow velocity achieved supersonic speed, residence time of fuel in the combustor is $\sim \mathrm{ms}$, and the mixing efficiency of fuel and air descends rapidly with the increasing of Mach number ${ }^{[1]}$. (2) In supersonic air flow, compression and stability of the mixed layer informed by mixing of fuel and air are very strong. At the same main inflow and density ratio, diffusion coefficient of compressible mixed layer is $1 / 3$ of incompressible one ${ }^{[2]}$. (3) Because the energy density of $\mathrm{H}_{2}$ is very low, hydrocarbon fuel should be used for realization of high specific impulse propulsion ${ }^{[3]}$.

Experimental method is usually used in the study on mixing of fuel and air of supersonic vehicle. However, for the limitation of costs, etc., superiority of numerical simulation becomes more and more outstanding gradually. Koji ${ }^{[4]}$ compared the reliability of tetrahedron mesh, optimized mesh and structured mesh with CFD software. Separated shear layer can be captured distinctly through using optimized mesh and structured mesh. For the capture of shocks, optimized mesh is more adaptive. Xiao ${ }^{[5]}$ computed the transverse jet flow field under supersonic inflow with SST turbulent model, and $\mathrm{N}-\mathrm{S}$ equations are solved with AUSMPW scheme. $\mathrm{He}^{[6]}$ added the component transport equations into the controlling equations, selected standard k-2 model as the turbulent model, then computed 2-D transverse side jet flow field under supersonic inflow. Sparkman ${ }^{[7]}$ optimized the fuel array spray design of scramjet with JET-PEN analysis software based on former experimental data.

In summary, as favorable supplement of experiments, study of numerical predictive method is very necessary, so as to provide references for engineering. Based on the needs, aimed at the problem of fuel jet and supersonic mixing in the combustor, transverse jet flow field under supersonic inflow is simulated numerically. In the further study, the method will be modified, and provide references for experiments.

\section{Computational method and conditions}

Computational zone and boundary conditions are shown in Figure 1. The number of mesh is 900, 000. Left edge is supersonic inflow, right edge is outflow, continuous line is no-slip solid surface, and imaginary line is symmetry boundary condition. The Mach number of inflow is 2.5 , static pressure is $5.8 \times 10^{4} \mathrm{~Pa}$, and static temperature is $130 \mathrm{~K}$. The diameter of jet is $2 \mathrm{~mm}$, total pressure of is $1 \mathrm{Mpa}$, and total temperature is $300 \mathrm{~K}$.

Corresponding author: $\underline{49225312 @ \text { qq.com }}$ 


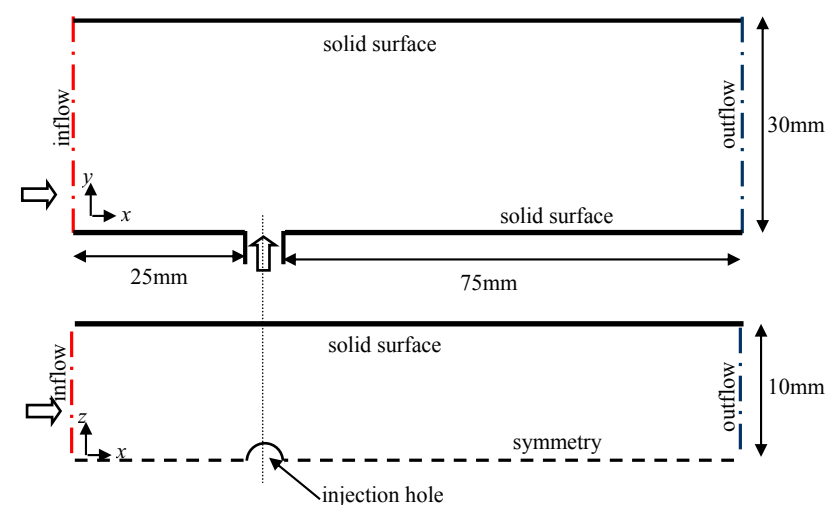

Figure 1. Computational zone and boundary conditions

Control equations of the CFD code programmed by us are 3-D unsteady compressible Reynolds average Navier-Stokes equations. Numerical method is implicit (dual-time step) finite volume method. Computation of inviscid flux is upwind scheme based on rotational Riemann resolver. MUSCL differential method is adopted, and the space accuracy achieved second-order. Second-order implicit scheme is used in time divergence, and the turbulent model is $k-\omega$.

\section{Results and Discussion}

\subsection{Simulated results}

Density gradients of equal spaced sections parallel to the symmetry are shown in Figure2. Separated shocks, trigeminal shocks and bow shocks can all be observed. From Figure 2, it is clear that with the increasing of $z$ value, the positions of separated shocks, trigeminal shocks and bow shocks moved backward. According to the space distributions of each section, separated shocks, trigeminal shocks and bow shocks are all around the jet, which agrees well with the 3-D configuration in Figure 3.

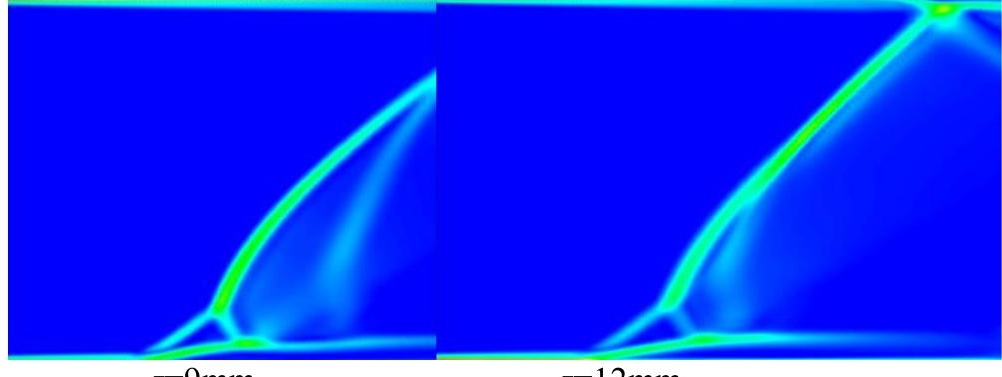

$$
z=9 \mathrm{~mm}
$$$$
z=12 \mathrm{~mm}
$$

Figure 2. Density gradients of equal spaced sections parallel to the symmetry

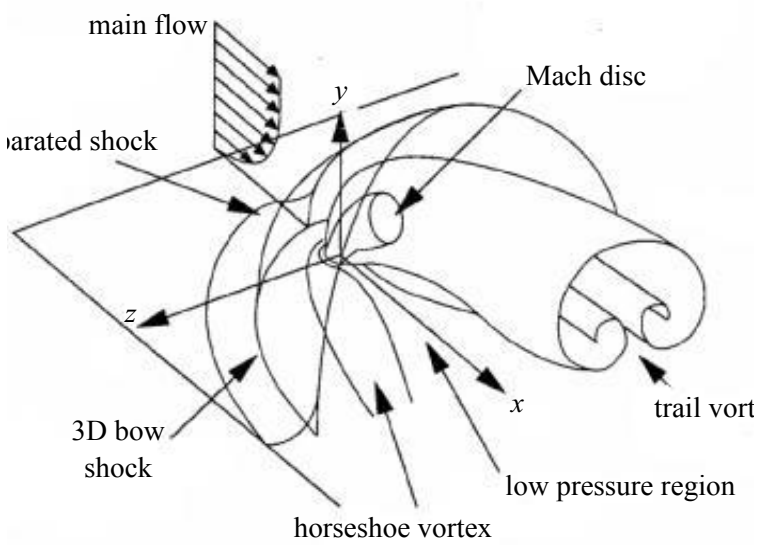

Figure 3. 3-D configuration of the transverse jet flow field under supersonic inflow ${ }^{[8]}$

\subsection{Comparison of references}

Simulated conditions of this paper and Cranfield University $^{[9]}$ are shown in Table 1. Mach number contours of both are shown in Figure 4 and Figure 5. It should be noticed that the simulated conditions is the same with that of experiments.

Table 1. Simulated conditions

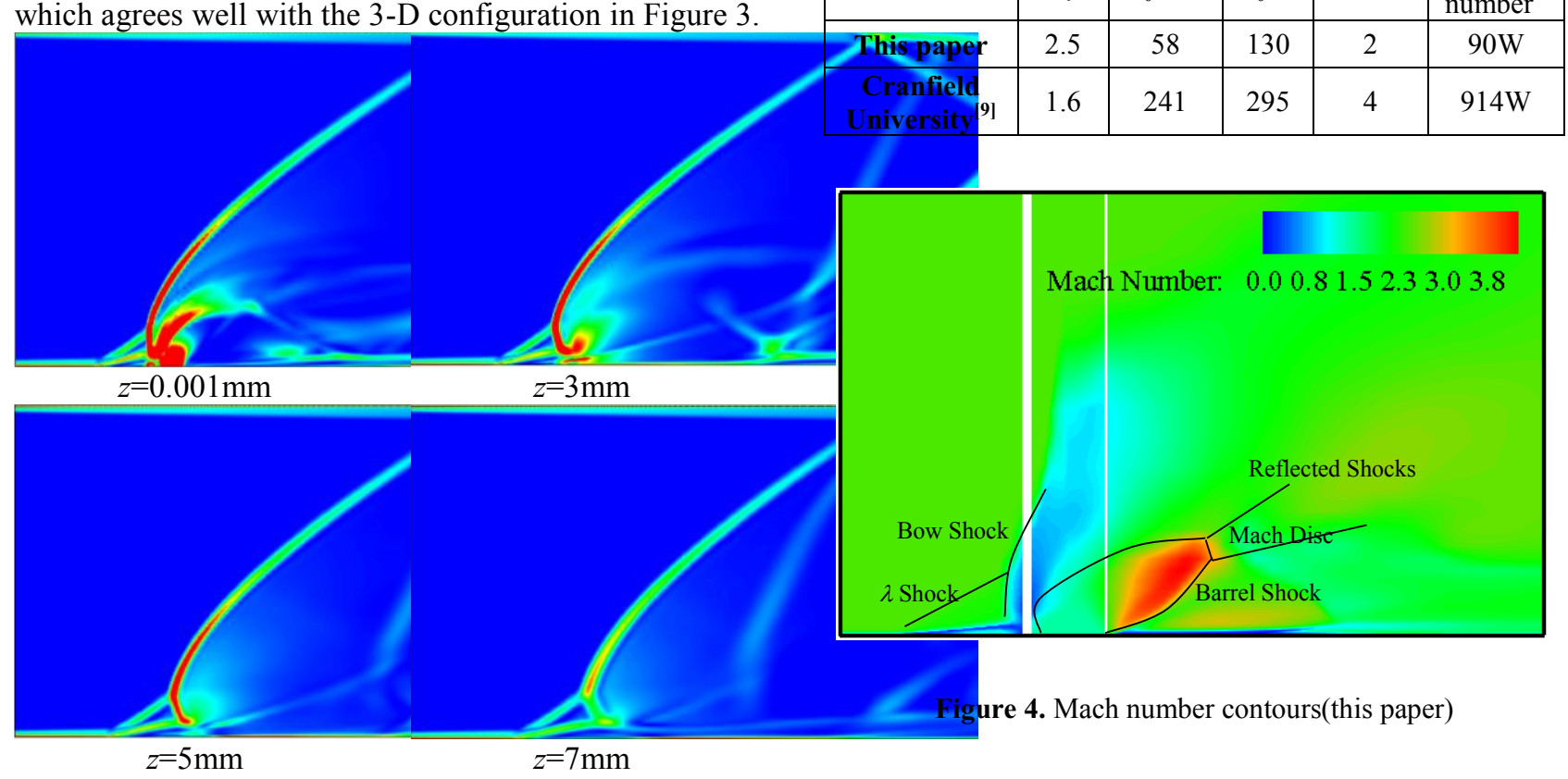




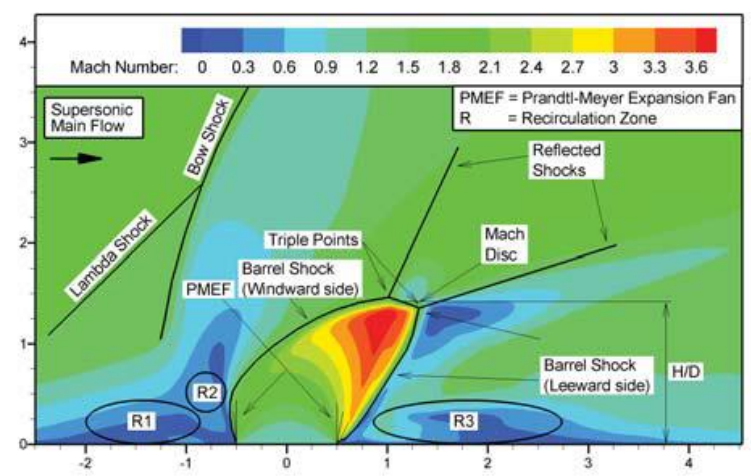

Figure 5. Mach number contours(Cranfield University) ${ }^{[9]}$

$\lambda$ shock, Mach disc and separated region can be observed in Figure 4, but the bow shock is not simulated ideally, and large scale eddies configuration is not shown. The reason is that the mesh is rare, especially the mesh far from the wall. There is no LES turbulent model in the program, too. Bow shock, Mach disc, $\lambda$ shock and separated region are all be shown in Figure 5. Although LES is adopted, large scale eddies is not simulated.

\subsection{Comparison of experiments}

Simulated result and schlieren photos of the flow field are shown in Figure 6. Figure 6 (a) is integral result of the density along $z$ axis. Figure 6 (b), (c) and (d) are photos of monochrome schlieren, continuous xenon lamp colored schlieren and strobe light colored schlieren respectively. The experimental model, inflow conditions and jet conditions are all same with that of simulation.

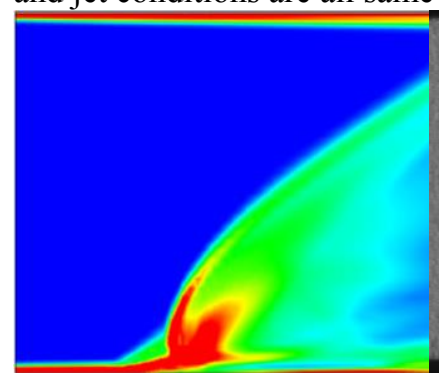

(a) simulated result

photo
phonom

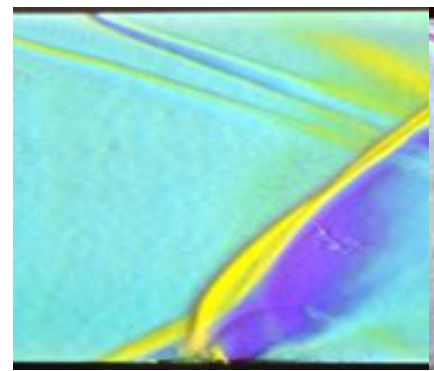

(c) continuous xenon lamp colored schlieren photo

Figure 6. Simulated result and schlieren photos of the flow field

From Figure 6) it can be observed that the simulated results agree well with that of experiments if the oblique shock induced by the rough upper wall is neglected. Mach disc, barred shock, bow shock, trigeminal shock and reflected shock can all be shown clearly. Compared with colored schlieren, information is less in monochrome schlieren, and the sensitivity is lower. Even so, if the cutting edge is deep enough, that is, the sensitivity of monochrome schlieren is high enough, barred shock and rear mixing zone can both be observed. Otherwise, distinction between bow shock and mixing layer boundary can be seen obviously through monochrome schlieren, while through the colored schlieren with high sensitivity, it is not easy to be districted. Photos taken by long time exposure can display wave configuration. Exposure time of several ns is suitable for the display of turbulence, especially the disordered situation of turbulence can be observed through colored schlieren.

\section{Conclusion}

From the comparison of research results, references and experiments, barred shock and Mach disc can be simulated, while $\lambda$ shock and back flow zone in transverse jet can be computed, too, which indicates that the computational method and scheme is suitable for research of this kind of problem. However, the deficiency is that detailed vortexes are not be displayed. The main reasons are: (1) the number of mesh is few; (2) there is no LES module in CFD code; (3) affection on transverse jet of boundary layer development is not considered. In the further study, modification should be made aimed at the above problems, so more accurate numerical predictive method would be provided.

\section{Acknowledgement}

This research was supported by the Natural Science Foundation of China (11372356, 11372357).The authors would like to acknowledge all discussions and technical help provided by the former and current members of the State Key Laboratory of Laser Propulsion and Application

\section{References}

1. John M, Seiner S M. Historical Survey on Enhanced Mixing in Scramjet Engines[R]. Journal of Propulsion and Power, 1998, 4(4): 1273-1286.

2. Georgiadis N J, Alexander J L D, Reshotko E. Investigation of a compressible turbulent mixing layer using a Hybrid RANS-LES method[R]. AIAA 2001-2563.

3. Law C K. Combustion Physics[D]. Cambridge University Press, 2006.

4. Koji M, Yoshiyuki K. Effect of Grid Resolutions and Injectant Species in Computations of Supersonic Mixing[R]. AIAA 2009-3946.

5. Xiao Yinli, Song Wenyan, Zhao Zhi. Numerical study of injection angle effect on fuel mixing characteristics in supersonic flow[J]. World Sci-tech R\&D, 2008, 30(4), 436-439. 
6. He Bo, Nie Wansheng, Feng Songjiang. Numerical simulation of underexpand heterogeneous lateral jet into supersonic air freestream[J]. Journal of the Academy of Equipment Command \& Technology, 2008, 19(3), 74-79.

7. Sparkman B T, Chrissis J W, Gruber M R, et al. Optimization of a Scramjet Fuel Injection Array: An Application of Mixed Variable Generalized Pattern Search With Kriging Surrogates[R]. AIAA 20085861.

8. Gruber M R, Nejad A S, Dutton J C. Circular and Elliptical Transverse Injection into a Supersonic Crossflow-The Role of Large-Scale Structure [R]. AIAA 1995-2150.

9. Rana Z A, Thornber B, Drikakis D. An ILES analysis of transverse jet injection in to supersonic cross-flow with synthetic turbulent boundary layer[R]. AIAA 2011-231. 